\title{
Assessment of epilepsy-related knowledge among medical students in ethiopia
}

\begin{abstract}
Background: The undergraduate medical program needs to include an epilepsy course that is introduced to the third-year medical students and taught in the fourth and fifth years of medical education. The approach to this course has been reinforced in different medical schools with the goal of demystifying neurology in general and epilepsy in particular, thus preparing the general physicians-in-training for better patient management.
\end{abstract}

Objective: Assess epilepsy-related knowledge among medical students in Addis Ababa University, Ethiopia.

Material and Method: Two hundred thirty fourth year medical students' knowledge was accessed by a self-administered questionnaire.

Results: All fourth year medical students were agreed to participate and compliance was indeed $100 \%$ with regard to completing and returning the questionnaire. "Seizure is an abnormal electrical discharge in the brain" was considered by $96.5 \%$ of respondents, while $20.0 \%$ thought it was a form of abnormal movement. The rank of causes of epilepsy given by respondents was head injury $(95.2 \%)$, brain tumors $(93.9 \%)$, and high fever $(90.0 \%)$ $57.0 \%$ of respondents thought epilepsy was curable disease. Most respondents $(87.4 \%)$ knew that epileptics should not be allowed to drive and $77.4 \%$ respond that epileptics should not work with machinery. Most respondents $(95.2 \%)$ would try to prevent injury during the episode.

Conclusion: The findings indicated that lecturers should emphasize on type of seizure, cause, consequences, and prognosis including management.

Keywords: epilepsy, knowledge, medical student, seizure
Volume 6 Issue I - 2017

\section{Seti Kelemework Belay, Dawit Kibru Worku \\ Addis Ababa University, Ethiopia}

Correspondence: Seti Kelemework Belay, Addis Ababa University, Black Lion Hospital,Addis Ababa, Ethiopia,Tel 251912101314, Email setibelay@gmail.com

Received: January 01, 1970 | Published: January 10, 2017
Abbreviations: AEDs, antiepileptic drugs; CNDs, chronic non-communicable diseases; GTCs, generalized tonic-clonic seizures; IRB, institutional review board

\section{Introduction}

Chronic non-communicable diseases (CNDs) are an increasingly important health care problem in developing countries, ${ }^{1}$ and neuropsychiatry disorders account for more than a quarter of the global burden of disease. ${ }^{2}$

Ethiopia has a population of about 95 million, $85 \%$ of whom live in rural areas. In a community based study performed in a rural population of 60,000 in central Ethiopia between 1986 and 1988, epilepsy was the most common cause of neurological disability with a prevalence of 5.2/1000. ${ }^{3}$ Another study performed in 1998 in a rural population of 25,000 in northwest Ethiopia found a very similar prevalence of 5.3/1000. ${ }^{4}$ Despite the availability, of Phenobarbitone, a cheap and effective treatment, more than $90 \%$ of patients living in rural areas remain untreated ${ }^{5}$. Potential reasons for this include cultural factors, lack of awareness of medical treatment and inaccessibility of medical services. ${ }^{6}$

There is a great degree of ignorance and unawareness associated with epilepsy amongst the medical as well as non- medical individuals at large. The metaphysical (i.e. supernatural) associations that are often linked with epileptics affect the quality of life of such patients, more than the affliction itself and also generate negative attitudes and prejudice on individuals with epilepsy. ${ }^{\text {? }}$

Young medical students are the future physicians of society and during their undergraduate period, they can be a source of health awareness and opinion formers to the general population. For this purpose, their knowledge must be assessed so they may play a pivotal role in society to educate the populace regarding various diseases. Most medical students are aware of the fact that stigmatizing epileptics is false and has no scientific grounds. ${ }^{8}$ Yet they encounter individuals who hold firm beliefs of supernatural associations with epileptics. As future health care providers, they should be trained appropriately to deal with situations which might put the patients' psychological conditions at stake due to social burden. ${ }^{9}$ Hence, prompt steps to spread awareness among the population include the proper attitude and understanding of medical professionals towards epileptic patients as well as counseling of common man so as to improve their approach towards epileptics because eradicating ignorant beliefs and practices is essential to improving the quality of life of these patients.

Health sector workers need to be conscious of their patients' beliefs about illness, and alternative treatment options that patients may choose. ${ }^{10}$ This need is even greater when it concerns widespread and stigmatizing diseases such as epilepsy. Epilepsy is a major public health problem in Ethiopia, with some areas having prevalence rates as high as $6 \% .{ }^{4}$ Furthermore, the human resources needed for optimal epilepsy care provision do not match the demand in developing countries like Ethiopia. ${ }^{11}$ Therefore, medical students and young physicians in Ethiopia need to be adequately educated about the beliefs and practices concerning epilepsy, given that perceptions are influenced by culture and traditional beliefs. An understanding of such beliefs and practices among medical students and young physicians themselves is an essential first step for the improvement of epilepsy education in medical schools in Ethiopia. Studies in Brazil ${ }^{12}$ and Scotland ${ }^{13}$ have concluded that increasing the knowledge and 
changing the attitudes of medical students concerning epilepsy could considerably improve the quality of life for patients.

The undergraduate medical program needs to include an epilepsy course that is introduced to the third-year medical students and taught in the fourth and fifth years of medical education. The approach to this course has been reinforced in different medical schools ${ }^{14,15}$ with the goal of demystifying neurology in general and epilepsy in particular, thus preparing the general physicians-in-training for better patient management. Results from this research will help to point the knowledge gap among medical students. It is crucial for medical lecturers to identify the knowledge level of the medical students in order to prepare appropriate lessons. In the present research, the authors plan to evaluate the knowledge of students. Upon knowing the level of knowledge, attention can be directed at developing a more effective presentation and lectures for the third-year and fourth-year students and better integration between the preclinical and clinical levels.

There is little information available in Ethiopia on the area of epilepsy and specially the management gap in our health system. Patients with epilepsy face a list of stigmas, disease related stigma also a key determinant of quality of life in patients with epilepsy. Assessing epilepsy-related knowledge among medical students is, therefore, critical to achieve the optimal care that is needed for patients with this disease.

An understanding of such beliefs and practices among medical students and young physicians themselves is an essential first step for the improvement of epilepsy education in medical schools in Ethiopia. Different previous surveys pointed a knowledge gap among medical students about epilepsy. ${ }^{8,12}$ We assumed that the same knowledge gap exist among Ethiopian medical students.

Therefore, we proposed an objective to determine an average estimate of medical students acquainted with epilepsy-related knowledge. We also tried to verify as to whether these students held a similar stigma towards epileptics as the general population.

\section{Materials and methods}

A Cross-sectional point prevalence survey was conducted on June 2016 in the college of health sciences, Addis Ababa University, Ethiopia. All fourth year medical students at Addis Ababa University were the source population. A total of two hundred thirty students were interviewed.

Students were included if they were clinical year two medical students, and informed consent granted for study participation. Students were excluded if they were or if they were unable to give consent. All students fulfilling the inclusion criteria from June 01, 2016 to June 30, 2016 in the study area was included. Clinical data was collected using a questionnaire in English. The questionnaire was based on items allied to epilepsy-related knowledge, consisting of 7 questions already applied in the previous survey. ${ }^{8}$ These include demographic data and detailed assessment of knowledge of epilepsy among medical students.

The researchers calculated the number of correct responses for each item (maximum $=50)$ : 1 ) what is a seizure?; ${ }^{2}$ ) what are the causes of epilepsy?; 3) what types of seizures are included?: ${ }^{10} 4$ ) is epilepsy curable?; ${ }^{5} 5$ ) for how long should antiepileptic drugs be taken ${ }^{5}$; 6) what are the consequences of seizures?: ${ }^{10}$ and, 7) how should acute seizures be managed?. ${ }^{10}$ Statistical analysis includes the percentage of correct responses and the means of the total scores.
Piloting of the questionnaire was done in a sample of ten students before the starting of data collection for the research. These students were not included in the study results. Findings from the pre-test were utilized in modifying questions on the standard questionnaire. Questions that were difficult for subjects to understand were reformulated and repiloted until answers were considered internally valid.

Interviews and data extraction was performed by the principal investigator. Data was checked manually and cleaned. Before processing the data was coded and cross checked for completeness. Analysis was performed using SPSS/PC version 20.0 software packages for statistical analysis (SPSS, INC, Chicago, IL). Descriptive summaries were employed to describe socio-demographic and clinical characteristics. Appropriate measures of central tendency, frequency distribution and cross tabulation were conducted. Odds ratios and $95 \%$ confidence intervals were calculated. A p value less than 0.05 were considered a statistically significant association between assessed variables. Due to the exploratory nature of our study we did not correct for multiple comparisons.

Protocol approvals were obtained from the ethical review Committee of the Department of Internal Medicine and the Institutional Review Board (IRB) and Research and Publication Committee of the College of Health Sciences of Addis Ababa University. Informed patient consent was sought before study enrollment. Students' data was deidentified during subsequent analysis and dissemination.

\section{Results and discussion}

All fourth year medical students were agreed to participate and compliance was indeed $100 \%$ with regard to completing and returning the questionnaire. Table 1 present percentage of answers to the questions and Table 2 presents the mean score for all of the knowledge questions.

Table I Frequency distributions of sociodemographic characteristics of study subjects, Addis Ababa University

\begin{tabular}{|c|c|c|}
\hline Variables & Number & Percent \\
\hline \multicolumn{3}{|l|}{ Age } \\
\hline$\leq 23$ & 160 & 69.6 \\
\hline$>23$ & 70 & 30.4 \\
\hline Mean/无D & $23.2 / 1.27$ & \\
\hline \multicolumn{3}{|l|}{ Gender } \\
\hline Female & 76 & 33 \\
\hline Male & 154 & 67 \\
\hline \multicolumn{3}{|l|}{ Religion } \\
\hline Orthodox Christian & 150 & 65.2 \\
\hline Protestant Christian & 36 & 15.7 \\
\hline Muslim & 30 & 13 \\
\hline Other & 14 & 6.1 \\
\hline \multicolumn{3}{|c|}{$\begin{array}{l}\text { Do you have a family member } \\
\text { affected by Epilepsy? }\end{array}$} \\
\hline Yes & 14 & 6.1 \\
\hline No & 216 & 93.9 \\
\hline \multicolumn{3}{|c|}{$\begin{array}{l}\text { Have ever witnessed a seizure } \\
\text { attack? }\end{array}$} \\
\hline Yes & 154 & 67 \\
\hline No & 76 & 33 \\
\hline
\end{tabular}


Table 2 Epilepsy-related knowledge among medical students, Addis Ababa University

\begin{tabular}{lll}
\hline Questionnaire & $\begin{array}{l}(\mathbf{n}=\mathbf{2 3 0}) \\
\text { Yes (Number) }\end{array}$ & Yes (Percent) \\
What do you think an epileptic attack & & \\
is? (Check all that you think apply) & & 96.5 \\
An abnormal electrical discharge in the brain & 222 & 2.6 \\
Demonic possession & 6 & 1.3 \\
Divine punishment & 3 & 20 \\
An abnormal movement & 46 &
\end{tabular}

What do you think causes epilepsy?

(Check all that you think apply)

\begin{tabular}{|c|c|c|}
\hline An evil sprit & II & 4.8 \\
\hline A head injury & 219 & 95.2 \\
\hline Brain tumor & 216 & 93.9 \\
\hline Divine punishment for reneging on a vow & 3 & 1.3 \\
\hline Sleep deprivation & 135 & 58.7 \\
\hline Alcohol withdrawal or heavy drink & 206 & 89.6 \\
\hline Stroke & 203 & 88.3 \\
\hline Genetic disease & 192 & 83.5 \\
\hline High fever & 207 & 90 \\
\hline Eating pork & 42 & 18.3 \\
\hline \multicolumn{3}{|l|}{$\begin{array}{l}\text { What are the types of seizures? (Check } \\
\text { all that you think apply) }\end{array}$} \\
\hline Tonic-clonic seizure & 226 & 98.3 \\
\hline Simple partial seizure & 222 & 96.5 \\
\hline Complex partial seizure & 216 & 93.9 \\
\hline Atonic seizure & 220 & 95.7 \\
\hline Absence seizure & 224 & 97.4 \\
\hline \multicolumn{3}{|l|}{ Do you think epilepsy can be cured? } \\
\hline Yes & 131 & 57 \\
\hline No & 99 & 43 \\
\hline \multicolumn{3}{|l|}{$\begin{array}{l}\text { How long antiepileptic drugs should be } \\
\text { taken? }\end{array}$} \\
\hline For life & 132 & 57.4 \\
\hline $2-5$ years & 83 & 36.1 \\
\hline Only on the full moon & 5 & 2.2 \\
\hline Only during an episode & 7 & 3 \\
\hline For 3-6 months & 3 & $\mathrm{I} .3$ \\
\hline \multicolumn{3}{|l|}{$\begin{array}{l}\text { What are the consequences of } \\
\text { epilepsy? (Check all that you think } \\
\text { apply) }\end{array}$} \\
\hline Should not allowed to drive motor vehicle & 201 & 87.4 \\
\hline No sexual intercourse & 10 & 4.3 \\
\hline Cannot get married & 4 & 1.7 \\
\hline Should not work with machinery & 178 & 77.4 \\
\hline Cannot get pregnant & 10 & 4.3 \\
\hline $\begin{array}{l}\text { Abruptly stop antiepileptic drugs during } \\
\text { pregnancy }\end{array}$ & 35 & 15.2 \\
\hline Not able to lactate & 10 & 4.3 \\
\hline Should not eat pork & 16 & 7 \\
\hline Must quit work & 7 & 3 \\
\hline Should not drink alcohol beverages & 127 & 55.2 \\
\hline \multicolumn{3}{|l|}{$\begin{array}{l}\text { What should be done during a seizure? } \\
\text { (Check all that you think apply) }\end{array}$} \\
\hline $\begin{array}{l}\text { Place the person in a semi-prone position to } \\
\text { prevent choking }\end{array}$ & 202 & 87.8 \\
\hline $\begin{array}{l}\text { Place something in the mouth to prevent } \\
\text { biting the tongue }\end{array}$ & 97 & 42.2 \\
\hline Give an antiepileptic drug during the episode & 135 & 58.7 \\
\hline $\begin{array}{l}\text { Restrain the person and perform chest } \\
\text { compressions }\end{array}$ & 26 & 11.3 \\
\hline Prevent injury during the episode & 219 & 95.2 \\
\hline
\end{tabular}

"Seizure is an abnormal electrical discharge in the brain" was considered by $96.5 \%$ of respondents, while $20.0 \%$ thought it was a form of abnormal movement. "Generalized tonic-clonic seizures (GTCs)" were the primary type of seizures recognized by $98.3 \%$ of respondents, while $97.4 \%$ were aware of "absence seizures". The rank of causes of epilepsy given by respondents was head injury $(95.2 \%)$, brain tumors $(93.9 \%)$, and high fever $(90.0 \%)$. Segment of medical students thought that eating pork (18.3\%), evil spirit $(4.8 \%)$, or punishment by the gods for reneging on vow $(1.3 \%)$ were causes of epilepsy. $57.0 \%$ of respondents thought epilepsy was curable disease. While about $57.4 \%, 36.1 \%$, and $3.0 \%$ thought epileptics needed to take antiepileptic drugs (AEDs) for life, 3-6 months, and only when there is an attack, respectively.

Most respondents (87.4\%) knew that epileptics should not allowed to drive and $77.4 \%$ respond that epileptics Should not work with machinery, while $4.3 \%$ thought patients should have not got pregnant or never have sexual intercourse. $98.3 \%$ thought epileptic patients should not be forbidden to marry. Most respondents (95.2\%) would try to prevent injury during the episode and $42.2 \%$ also would try to put an object into the mouth to prevent the tongue from being bitten and $11.3 \%$ would try to restrained the patient and administer chest compression, and $58.7 \%$ would give AEDs during the attack.

The knowledge scores included seven items and the mean score was about $74.9 \%(37.46 / 50.00)$. Knowledge about "What are types of seizures?" achieved the highest scores (96.35\%), while "How to manage acute seizures?" scores were answered correctly by $80.3 \%$ $(8.03 / 10)$ of respondents. The different causes of seizures had the lowest scores $44 \%(2.2 / 5)$. In the present study, the knowledge of fourth year medical students' concerning epilepsy revealed a $60 \%$ total mean score of knowledge compared with $75 \%$ among fifth-year medical students(1) and $47.3 \%$ of first-year medical students. ${ }^{1}$ Senior medical students had the best level of familiarity and knowledge. ${ }^{2}$

Most (96.5\%) of the respondents knew that an epileptic attack is an abnormal electrical discharge in the brain. But $2.6 \%$ and $1.3 \%$ thought it was the result of demonic possession and divine punishment, respectively. By contrast, $30 \%$ of rural Ethiopians ${ }^{3}$ and $60 \%$ of epileptics and their parents thought it had a demonic origin. ${ }^{16}$ While none thought it is caused by any supernatural cause in a similar study from Thailand. ${ }^{8}$ A significant proportion of respondents in other studies harbored supernatural associations as predisposing factors ${ }^{17,18}$ despite medical evidence showing that epilepsy arises from a transient dysfunction in the brain. ${ }^{19}$

Since the present findings reflect medical students' understanding of epilepsy, it is of concern that $20.0 \%$ think an epileptic attack could simply be abnormal movement. In contrast, in Thailand study only $15.5 \%$ think an epileptic attack could simply be abnormal movement. Knowledge of the classification of seizure types revealed that $98.3 \%$ of medical students recognized GTCs, similarly in the Thailand study it was reported to be $95.5 \%{ }^{8}$ While $97.4 \%$ and $96.5 \%$ were aware of "absence seizures" and "simple partial seizures". In contrast, in the Thailand study most did not recognize absence seizures $(88.2 \%)$ or absence seizures $(66.4 \%),{ }^{8}$ suggesting our medical students had a good fund of knowledge about type of epilepsy.

Regarding the causes of epilepsy, segment of our medical students thought that eating pork $(18.3 \%)$, evil spirit $(4.8 \%)$, or punishment by the gods for reneging on vow $(1.3 \%)$ were causes of epilepsy. In contrast, in Thailand study none of the medical students responded these as a cause of seizure. ${ }^{8} 11.7 \%, 10.4 \%$, and $41.3 \%$ of the students from the present study think stroke, alcohol withdrawal or heavy drinking and sleep deprivation, respectively. In contrast, a large 
number of the students did not realize that stroke (80.9\%), alcohol withdrawal or heavy drinking (69.1\%) and sleep deprivation $(92.7 \%)$ could cause or induce epilepsy in the Thailand study. ${ }^{8} 90.0 \%$ of them knew high fever as a cause of seizure in the current study while only $67.3 \%$ in the Thailand study, ${ }^{8}$ Interestingly, $83.5 \%$ of the students thought epilepsy was genetic in origin, similar to a report from a Malaysian study $(67 \%)^{19}$ and the Thailand study $(50.0 \%),{ }^{8}$ although no more than $1-2 \%$ of cases are due to genetic disorders. ${ }^{18}$

Regarding epilepsy management, $57.4 \%, 36.1 \%$, and $3.0 \%$ of medical students thought epileptics should take AEDs for 2 to 5 years, only during an attack and 3 to 6 months, respectively. Similarly, $48.3 \%, 13.6 \%$ and $4.5 \%$ of medical students in the Thailand study responded epileptics should take AEDs for 2 to 5 years, only during an attack and 3 to 6 months, respectively. ${ }^{8}$ Although all of the respondents had learned about epilepsy in the preclinical and clinical setting, only $57.0 \%$ knew that epilepsy can be cured, but more than the $46.3 \%$ reported by the Malaysian study ${ }^{19}$ and $28.2 \%$ by the Thailand study. ${ }^{8}$ Even though our students knew better about the prognosis of epilepsy than the two studies, a significant of our students $(43.0 \%)$ retain misconceptions.

Concerning the consequences of epilepsy, some respondents had incorrect knowledge as they thought patients should have not got pregnant or never have sexual intercourse (4.3\%). This is more than the Thailand study, $1.8 \%$ thought patients should have not got pregnant or never have sexual intercourse. ${ }^{8}$ Most respondents (87.4\%) knew that epileptics should not be allowed to drive and $77.4 \%$ respond that epileptics should not work with machinery. This is comparable with the Thailand study, $80.0 \%$ respondents knew that epileptics should

Table 3 Score of epilepsy-related knowledge in each item

\begin{tabular}{lll}
\hline Items & $\begin{array}{l}\text { Present Study } \\
\text { Score (mean + SD) }\end{array}$ & $\begin{array}{l}\text { The Thailand study (8) } \\
\text { Score(mean + SD) }\end{array}$ \\
\hline What is seizure? (5) & $4.587 \pm I .148$ & $4.59+1.38$ \\
What are causes of epilepsy? (5) & $2.2 \pm I .038$ & $2.4 I+0.96$ \\
What are types of seizures? (I0) & $9.635 \pm I .228$ & $4.30+2.12$ \\
Is seizure a curable disease? (5) & $2.848 \pm 2.48 \mathrm{I}$ & $3.59+2.26$ \\
How long to take antiepileptic drugs? (5) & $2.87 \pm 2.478$ & $2.36+2.5 \mathrm{I}$ \\
What is/are consequence of seizures? (I0) & $7.287 \pm 3.177$ & $5.17+2.39$ \\
How to manage acute seizures? (I0) & $8.03 \pm 2.194$ & $7.18+2.54$ \\
Total (50) & $37.457 \pm 3.40 I 1$ & $29.60+7.54$ \\
\hline
\end{tabular}

\section{Conclusion}

The present study demonstrated that epilepsy-related knowledge among our medical students was incomplete and some are alarming. The content of the third and fourth year medical students' epilepsy lecture should emphasize the types of seizure, causes of epilepsy, consequences, general and acute management. The outcome would be both better knowledge and improved physician-patient relations. A follow-up study with the presented questionnaire administered by the same group of students after attending the lecture will be done to assess for the degree of improvement in their knowledge and practice regarding epilepsy.

\section{Acknowledgments}

None.

\section{Funding}

None. not be allowed to drive and $74.5 \%$ knew that the people with epilepsy should avoid working with machinery. ${ }^{8}$

Regarding management of epileptics during episode, $42.2 \%$, $11.3 \%$ and $58.7 \%$ of respondents indicated incorrect protocols: placing an object in the mouth to prevent the tongue from being bitten, restraining the patient and/or performing chest compressions, and giving AEDs during an episode, respectively. The present findings are consistent with studies in China $^{20}$ and the Thailand study ${ }^{8}$ except "giving AEDs during an episode" which is more in the present study.

Most medical students $(95.2 \%)$ in the present study would try to prevent injury during the episode which is significantly more than the Thailand study (50.9\%) (8). $42.2 \%$ also would try to put an object into the mouth to prevent the tongue from being bitten, while in a Sri Lanka study $64 \%$ of respondents recommended placing a piece of wood in the mouth during a seizure. ${ }^{17} 11.3 \%$ would try to restrained the patient and administer chest compression, and 58.7\% would give AEDs during the attack. In contrast, in the Thailand study $20.0 \%$ would try to restrained the patient and administer chest compression, or give AEDs during the attack. ${ }^{8}$

Most medical students $(74.9 \%)$ in the present study had a mean score of epilepsy-related knowledge of $74.9 \%$, while in the Thailand study, it's $60 \%$. In another study conducted among school teachers in Thailand - it revealed that mean score of epilepsy-related knowledge is only $50 \%{ }^{20}$ (Table 3 ). The difference among the mean scores of epilepsy-related knowledge in the present study and the Thailand study is not significant with a t-value of -0.37526 and a p-value of 0.356546 . Thus, the medical school must also publicly disseminate correct knowledge and first aid management of epilepsy.

\section{Conflicts of interest}

None.

\section{References}

1. Unwin N, Mugusi F, Aspray T, et al. Tackling the emerging pandemic of non-communicable diseases in Sub-Saharan Africa: The essential NCD health intervention project. Public health. 1999;113(3):141-146.

2. Pal DK, Nandy S, Sander JWAS. Towards a coherent public health analysis for epilepsy. Lancet. 1999;353(9167):1817-1818.

3. Tekle-Haimanot R, Abebe M, Gebre-Mariam A, et al. Community based study of neurological disorders in rural central Ethiopia. Neuroepidemiology. 1990;9(5):263-277.

4. Berhanu S, Alemu S, Prevett M, et al. Primary care treatment of epilepsy in rural Ethiopia: causes of default from follow-up. Seizure. 2009;18(2):100-103.

5. Tekle-Haimanot R, Forsgren $\mathrm{L}$, Abebe $\mathrm{M}$, et al. Clinical and electroencephalographic characteristics of epilepsy in rural Ethiopia: a community based study. Epilepsy Research. 1990; 7(3):230-239. 
6. Tekle-Haimanot R, Forsgren L, Ekstedt J. Incidence of epilepsy in rura central Ethiopia. Epilepsia. 1997;38(5):541-546.

7. Jacoby A. Stigma, epilepsy, and quality of life. Epilepsy \& Behavior. 2002;3(6S2):10-20.

8. Tiamkao S, Tiamkao S, Auevitchayapat N, et al. Basic knowledge of epilepsy among medical students. J Med Assoc Thai. 2007;90(11):22712276 .

9. Thomas, SanjeevV, Aparna Nair. Confronting The Stigma Of Epilepsy. Ann Indian Acad Neurol. 2011;14(3):158-163.

10. Sands H, Zalkind SS. Effects of educational campaigns to change employer attitudes towards hiring epileptics. Epilepsia. 1972;13(1):87-96

11. World Health Organisation (WHO). World Federation of Neurology Neurology Atlas: Country resources for neurological disorders. World Health Organization Programme for Neurological Diseases and Neuroscience, Department of Mental Health and Substance Abuse. Geneva. 2004.

12. Fonseca LC, Tedrus GM, Costa AC, et al. Knowledge and attitude towards epilepsy among health area students. Arq Neuropsiquiatr. 2001;62(4):1068-1073.

13. Mason C, Fenton GW, Jamieson M. Teaching medical students about epilepsy. Epilepsia. 1990; 31(1):95-100.
14. Njamnshi AK. Medical curriculum development: a proposal based on a teacher's reflections. Health Sci Dis. 2000;2:26-29.

15. Njamnshi AK. Medical curriculum development: the educational theory underlying the proposal. Health Sci Dis. 2000;3:54-61.

16. Levy LF. Epilepsy in Rhodesia, Zambia and Malawi. Afr J Med Sci. 1970;1(3):291-303.

17. Senanayake N, Abeykoon P. Epilepsy in Sri Lanka: public awareness and attitudes. J Trop Med Hyg. 1984;87(2):61-66.

18. Ismail $\mathrm{H}$, Wright $\mathrm{J}$, Rhodes $\mathrm{P}$, et al. South Asians and epilepsy: exploring health experiences, needs and beliefs of communities in the north of England. Seizure. 2005;14(7):497-503.

19. Ab Rahman AF. Awareness and knowledge of epilepsy among students in a Malaysian university. Seizure. 2005;14(8):593-596.

20. Fong CY, Hung A. Public awareness, attitude, and understanding of epilepsy in Hong Kong Special Administrative Region, China. Epilepsia. 2002;43(3):311-316

21. Tiamkao S, Aaauevitchayapat N, Arunpongpaisal S, et al. Knowledge of epilepsy among teachers in Khon Kaen Province, Thailand. J Med Assoc Thai. 2005;88(12):1802-1808. 